\title{
Characteristics of community-based exercise programs for community-dwelling older adults in rural/regional areas: a scoping review
}

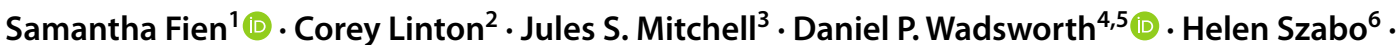 \\ Christopher D. Askew ${ }^{2,5}$ (D) Mia A. Schaumberg ${ }^{2,5,7}$ (D)
}

Received: 16 December 2021 / Accepted: 17 January 2022 / Published online: 12 February 2022

(c) The Author(s) 2022

\begin{abstract}
Community-based exercise programs for older adults play a potentially important role in mitigating the decline in functional outcomes, body composition, psychosocial outcomes, and cardiovascular health outcomes that commonly occurs with advancing age. There is a limited understanding of the characteristics and effectiveness of community-based exercise programs, particularly when those programs are offered outside metropolitan areas. Rural/regional settings face unique challenges, such as limited access to equipment/resources, transportation, and services, as well as significant costs to run programs. The objective of this scoping review was to characterise studies in the field that have aimed to implement community-based programs in settings identified as rural / regional. A secondary aim was to establish guidance for future exercise programs in this setting and highlight future research directions. A total of 12 studies were conducted in settings identified as rural/regional areas in various countries across the world were included. Of the included studies, five were randomised controlled trials. The majority of included studies reported on functional outcomes $(83 \%)$ and psychosocial outcomes (75\%), yet only $42 \%$ reported body composition, $17 \%$ reported cardiovascular health and $17 \%$ reported dietary outcomes. Low male representation was observed, with women outnumbering men in 7 of 12 studies. There was also minimal investigation of qualitative outcomes in existing community-based exercise programs in rural/regional settings, presenting a key gap for future research to address. Study Protocol: https://osf.io/txpm3/. Date of registration: 20 July 2020.
\end{abstract}

Keywords Older adult $\cdot$ Physical function $\cdot$ Psychosocial health $\cdot$ Regional/rural

\section{Introduction}

Samantha Fien

s.fien@cqu.edu.au

1 School of Health, Medical and Applied Sciences, Central Queensland University, Mackay, Australia

2 School of Health and Behavioural Sciences, University of the Sunshine Coast, Sippy Downs, Australia

3 Thompson Institute, University of the Sunshine Coast, Birtinya, Australia

4 School of Nursing, Midwifery and Paramedicine, University of the Sunshine Coast, Sippy Downs, Australia

5 Sunshine Coast Health Institute, Sunshine Coast Hospital and Health Service, Birtinya, Australia

6 Sunshine Coast Council, Birtinya, Australia

7 School of Human Movement and Nutrition Sciences, The University of Queensland, Brisbane, Australia
Ageing is associated with an increased risk of chronic disease, profoundly affecting the independence, mobility, and quality of life of older adults [1]. Globally, the most recent data estimate between 60 and $75 \%$ of adults over the age of 65 will be diagnosed with one or more chronic diseases [2-5]. Aside from the adverse effects on individual physical and cognitive functions, at the societal level, the financial burden of chronic disease management and treatment is profound, as evidenced by the United States expenditure constituting approximately $17 \%$ of GDP [6]. With the ageing population worldwide expected to double by 2050 [7], this issue will undoubtedly be compounded. This presents an unsustainable pressure on expenditure within the health system, that if to be mitigated, requires immediate attention. Interestingly, according to the models of Scott et al. [5], interventions targeted at reducing the impact of ageing 
on functional decline and dependence, have the potential to save US \$38 trillion globally in 1 year [5].

Similar to many health-related issues, the challenges associated with an ageing population and chronic disease burden are likely to be exacerbated in rural/regional areas owing to the limited access to healthcare professionals and resources $[8,9]$. What-is-more, exercise interventions can have profound results at an individual and societal level, for example in maintaining cognition, physical function, and wellbeing such that older adults may be able to lead a healthier, fulfilling, and productive life whilst remaining active contributors to their community [10-12]. Accordingly, there is a significant need to develop and implement more effective, and sustainable lifestyle interventions to manage, treat, and most desirably, prevent chronic diseases or their progression. Exercise presents as one of the most easily modifiable lifestyle factors with considerable evidence demonstrating its efficacy in mitigating the rate of age-related decline in physical, mental, and psychosocial health [13]. Whilst these positive benefits of exercise are well-documented [14], physical inactivity has remained high in the developed world [15], particularly in older adults [16].

Community-based group exercise programs are one approach that aim to encourage, enable, and engage older adults to participate in regular, appropriate, and healthpromoting exercise. Importantly, these programs have the added potential to stimulate social engagement amongst members of the community; a factor known to improve health outcomes in older age, in and of itself [17-19]. This is achieved through maximising accessibility (sessions are held at community halls and local gyms); and financial affordability (sessions are free or low-cost). Previous systematic reviews of community-based exercise programs for older adults have shown that such programs can promote regular physical activity and improve general health outcomes [4, 20, 21]. In particular, improvements in mobility and physical function outcomes, and how they relate to reductions in frailty and falls risk, have consistently been reported as a common focus in the available research $[4,20]$.

A systematic review of community-based exercise programs in specifically rural/regional areas reported similar findings [22]. All seven studies included in that review incorporated low-to-moderate intensity exercise (e.g., tai-chi, balance, aquatics, yoga), and this led to positive changes in various physical and functional outcomes (e.g., balance, walking, strength, aerobic capacity) in five studies [23-27]. Notably, one study included a qualitative interview measure to assess perceived changes following the exercise program [27], with improvements in mental outlook reported. Despite the depth of existing literature, significant variability in study design, population characteristics, exercise program (i.e., type, duration, intensity, delivery), location, adherence levels, and outcome measures have been identified as key limitations [4, 22, 28]. Furthermore, because most research has focused on physical and functional outcomes, there is little known about the effect that rural/regional community-based exercise programs can have on perceived psychological and psychosocial outcomes in older adults.

Consequently, the current understanding of what factors influence the effectiveness, acceptability, and successful implementation of community-based exercise programs in older adults living in rural/regional areas is limited. The objective of this scoping review is to identify and summarise the characteristics and outcomes of existing community-based exercise programs for community-dwelling older adults living in rural/regional areas. In doing so, this will provide guidance to existing and future programs, and highlight future research directions [29].

This scoping review addresses the following questions:

1. What are the aims and characteristics (exercise modality, delivery mode, duration, and supervision) of community-based exercise programs in settings that are identified as rural/regional areas?

2. What are the demographics and health status of older adults participating in community-based exercise programs in settings that are identified as rural/regional areas?

3. What types of outcomes (primary or secondary) have been reported?

4. What factors/variables are associated with successful rural/regional community-based exercise programs?

Addressing these aims will support the development and implementation of resources and evidence-based approaches to support the successful implementation and evaluation of community-based programs for older adults residing in rural/regional areas.

\section{Methods}

\section{Protocol and registration}

Scoping review methodology was chosen as it is the most appropriate method to identify key characteristics related to the topic of investigation: community-based exercise programs for community-dwelling older adults living in rural/ regional areas [30]. This review followed the Joanna Briggs Institute (JBI) Scoping Review Methodology [31] and is reported according to the guidelines outlined in the PRISMA Extension for Scoping Reviews (PRISMA-ScR) [32]. Prior to commencing this review, the research team developed and 
published a scoping review protocol available at (https://osf. io/txpm3/). The protocol described the planned approach and full methods for this scoping review, and so these are described briefly in this paper.

\section{Eligibility criteria}

Included studies reported an evaluation / assessment of community-based exercise programs in settings identified as rural/regional areas with older adults, aged 65 years or older, which measured one, or a combination of, physical, psychological, or qualitative outcomes. There were no restrictions for the exercise programs in terms of delivery with the inclusion criteria being programs that targeted independently living older adults in settings identified as rural/regional areas. Given our focus on healthy older adults, studies that specifically targeted the treatment or rehabilitation of a diagnosed disease, condition, or injury (e.g., dementia, stroke, cancer, diabetes mellitus, and osteoarthritis) were excluded. No publication date restriction was applied, however, citations generated were limited to English studies. The study designs included were experimental (randomised controlled trials (RCTs), quasi-RCTs, non-RCTs), quasi-experimental (controlled before after, interrupted time series), and observational (cohort, case control, cross-sectional). Qualitative study designs (for example case study designs) as well as reviews, grey literature (for example dissertations and government reports), text, and opinion papers were included.

\section{Information sources and literature search}

A comprehensive literature search was conducted, which was not time-limited, in Cochrane Library (Wiley), PubMed (NLM), Scopus (Elsevier), and Web of Science (Clarivate). The search terms included community-based, independent, older adults, over 65, exercise, physical activity, rural, and regional. For studies that did not explicitly stipulate if the location was rural/regional, they were excluded from this review. To ensure a more comprehensive search, additional terms were incorporated to target rural/regional locations (e.g., semi-urban, non-metro, county). All search strategies, both initial and revised, for the other databases are available from the corresponding author upon request. A secondary search of the reference lists of the 12 included studies was carried out by two members of the team (JM and SF) to identify any additional studies.

\section{Study selection process}

A preliminary screen of 25 selected studies was conducted to establish a level of agreement in accordance with the standard set by JBI [31], and to flag any issues with the inclusion/exclusion criteria. As the level of agreement was greater than $90 \%$, the reviewers completed screening for the remaining studies according to the eligibility criteria outlined in the protocol. Studies were screened for inclusion through a two-step process; the first step screening title/ abstract and the second step assessing full-text articles, with any disputes between the two reviewers (JM and SF) resolved through discussion, or failing this, using an independent third reviewer (DC).

The following information was extracted: author, year and country of publishing, study design, study aim, follow-up timepoints, inclusion of a rural/regional definition, location of program, inclusion of a focus group, sample size, female percentage, reporting of chronic diseases in the sample, intervention duration, frequency of sessions, duration of session, exercise type, specific exercises, delivery mode, supervision and if that included a health professional, comparison/control group, adherence and influence of adherence reported. The main outcomes identified were extracted in tabular format: functional outcomes, body composition, psychosocial outcomes, cardiovascular health, and dietary outcomes.

\section{Results}

\section{Literature search}

From the database and grey literature search, 1814 citations were generated. Following duplicate removal, title/abstract and full-text screening against the eligibility criteria, 12 articles were included in the final review. A breakdown of the search results, duplicate removal, and reasons for study exclusion are reported in Fig. 1.

\section{Terminology and definition of rural/regional}

Due to there being no conclusive definition for rural/regional locations for this review, rural/regional was defined as follows: (1) the article needed to specifically include the terminology "rural/regional/other related term location (i.e., specify that the study was not conducted in a major city centre)". The characteristics of each study location can be found in Table 1. The used term to describe the location was rural (100\%). No studies provided a definition of what constituted a regional area. Most studies (58\%) reported where the delivery of the exercise intervention took: three in a community hall / centre; one at social club; one in the local gym; one in the village; one at home. However, five studies did not report the details. 


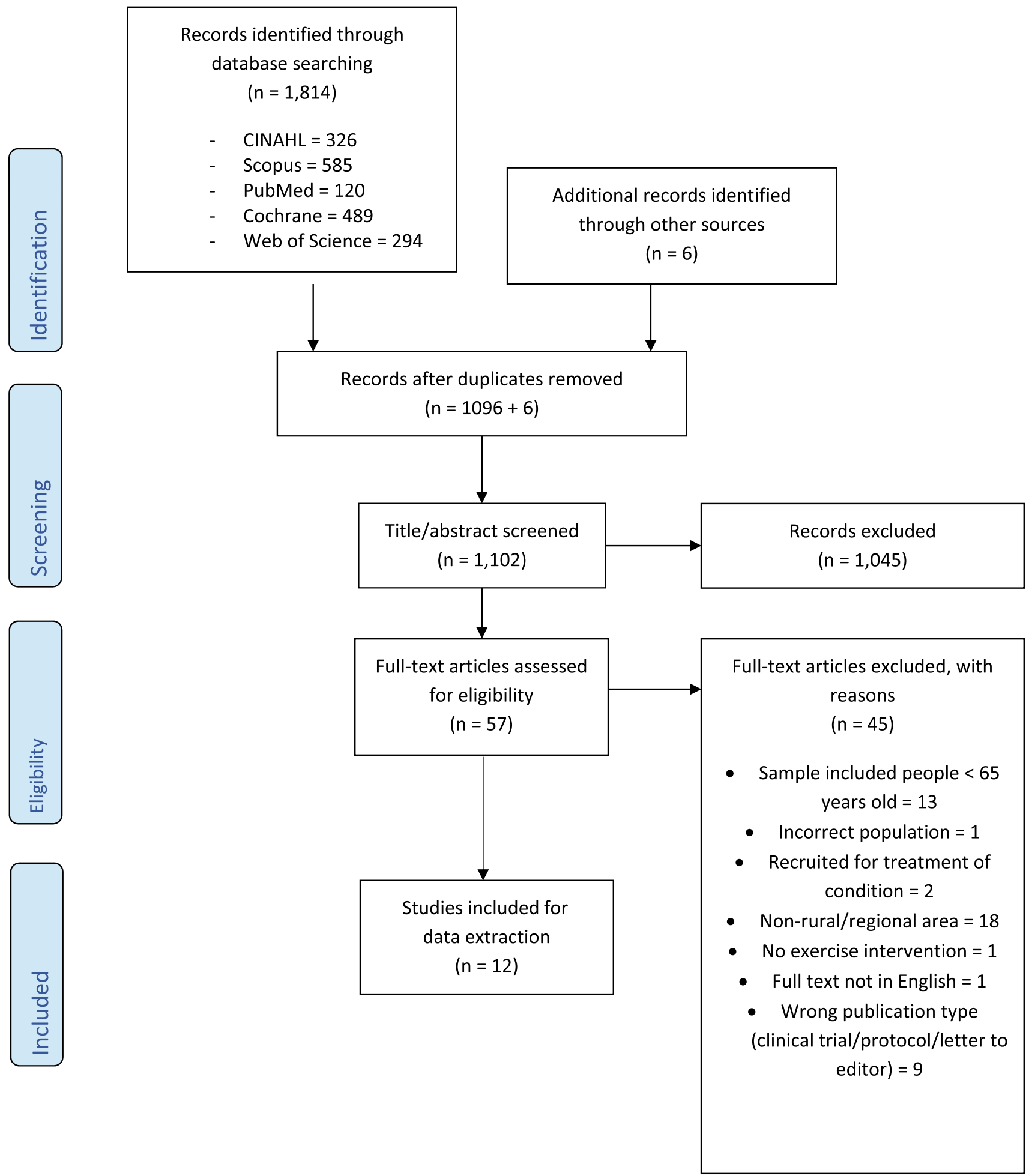

Fig. 1 PRISMA flow diagram describing record inclusion through different stages of screening; includes number of records identified, included and excluded, and the reasons for exclusion 


\section{Study characteristics}

Studies were published between 1996 and 2018, and were conducted in both western and non-western cultures. A large proportion of studies were conducted in the United States of America $(n=4,33 \%)$, Japan $(n=3,25 \%)$, and Taiwan, $(\mathrm{n}=2,16 \%)$, with the remaining locations consisting of Italy $(n=1,8.3 \%)$, Spain $(n=1,8.3 \%)$, and South Korea $(n=1,8.3 \%)$. Four studies were randomised controlled trials [37-39, 42] and seven were quasi-experimental studies [33-36, 40, 41, 43]. There were no observational or qualitative studies included. A further summary of the study characteristics can be found in Table 1 .

\section{Population characteristics}

The characteristics of the sample population for each study can be found in Table 2. Across all studies, the age of participants ranged from 65 to 90 years, with a mean of 74.73 $(\mathrm{SD}=5.35)$. The sample sizes ranged from 22 to 520 participants. Females constituted a larger portion of the sample size in ten studies: with female sample percentage below $50 \%$ in two studies [34, 38]. All included participants (including the comparison group) were relatively healthy (i.e., not requiring a carer, currently hospitalised, or specifically recruited for rehabilitation or treatment) and living independently within the local community.

\section{Exercise intervention characteristics}

The characteristics for each exercise intervention are presented in Tables 3 and 4.

Exercise type: Significant variations in type, delivery and duration of exercise were noted across the included studies. A total of six studies offered only a single exercise type [33-36, 38, 40], with six studies offering two or more exercise types. Of the studies with a single exercise type, square stepping (two studies), tai-chi (two studies) and aerobic training (two studies) were commonly reported followed by walking (one study), endurance exercise (one study). Of the multi-component exercise programs, two studies included combined balance/strength exercise, two studies combined aerobic/balance (with one being exergame), one fall-risk nutrition and hazard education, combined with strength, coordination, balance, mobility, and one study combined strength, balance, and flexibility exercises.

Exercise delivery: In most studies (10/12), exercise was the sole intervention. Three studies incorporated exercise as part of a multi-component intervention, alongside nutrition, counselling, and health seminars [27, 41-43]. Nearly all (11/12) exercise interventions were delivered in-person; of these, all 11 studies offered group exercise classes, and no studies offered individual exercise classes. Online, one-on-one exercise interventions were offered in two studies. The exercise was supervised in $83.3 \%$ of studies, with only $33.3 \%$ of the supervisors were universitytrained exercise professionals (e.g., exercise physiologists, sports scientists).

Exercise duration: Program duration ranged from 8 to 56 weeks, with the vast majority $(61.1 \%)$ between 8 to 12 weeks in duration. Exercise sessions were offered between 1 and 3 times per week, ranging from 15 to 120 min per session. Fifteen studies included pre- and postprogram assessments, and three reported at least one follow-up assessment. Follow-up time-points ranged from weekly to 56 weeks.

Adherence: Program adherence was recorded in 13 studies. Of these, adherence greater than $80 \%$ was reported in $46 \%$ of studies $(6 / 13)$, whilst three studies reported adherence below $60 \%$. Three studies identified factors that influenced adherence, including injury (50\%), physical inability to get to sites (25\%) and time constraints (25\%). Self-efficacy to participate in physical activity [41], and reduced cost $[33,43]$, were reported to influence adherence. In addition, the capacity to have individualised programs and education on self-management techniques [33]; a mix of age groups [37]. Notably, in one study that utilised a wearable device to promote physical activity, simplicity, ease of use, battery life, and affordability predicted high adherence [33].

Qualitative data: No qualitative data were reported for any of the included studies.

Control groups: Whilst the majority of the control group interventions had no details regarding the delivery mode of the intervention $(n=10,55.56 \%)$, the remainder were a combination of in-person group sessions $(n=5$, $27.8 \%)$, individual $(n=1,5.56 \%)$, at-home $(n=1,5.56 \%)$, and external—via wearable device $(n=1,5.56 \%)$.

\section{Outcome measures}

All outcome measures are summarised in Table 5. A total of 15 studies included physical function measures, with psychosocial measures reported in only 9 studies. Physical function measures included: single leg balance (4/12 studies), 30-s sitto-stand (2/12 studies), and timed up-and-go (7/12 studies). In addition, physical activity was measured with step count (6/12 studies). Fear of falling was the main psychosocial outcome measured, being reported in two studies. Cognition was only assessed in three studies (via the Mini-Mental State Examination (MMSE)). No studies employed focus groups as a means of obtaining qualitative measures. Total fat mass was the main measurement of body composition (3/12 studies) and only two studies reported outcomes for cardiovascular health. Only two 


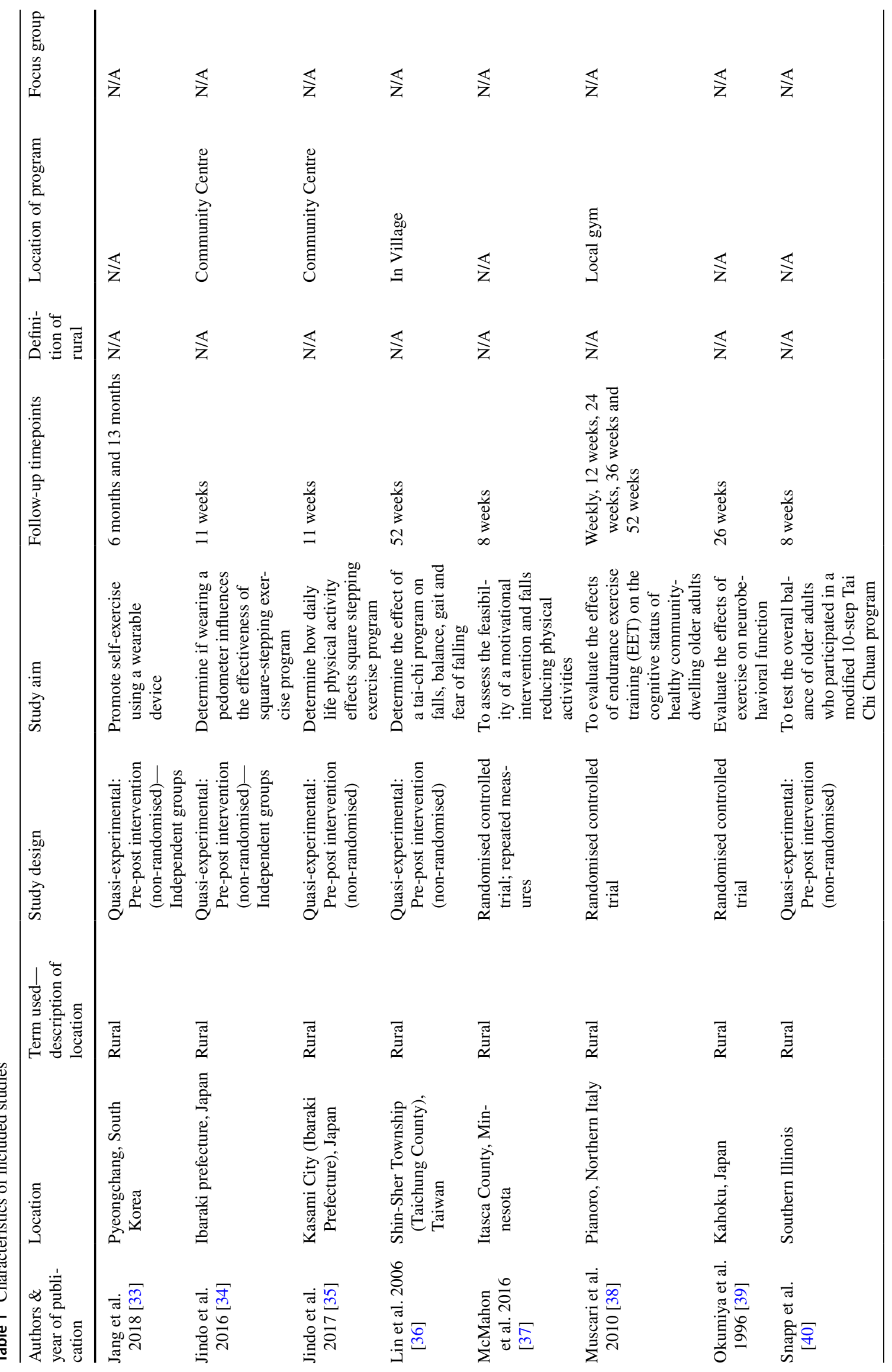




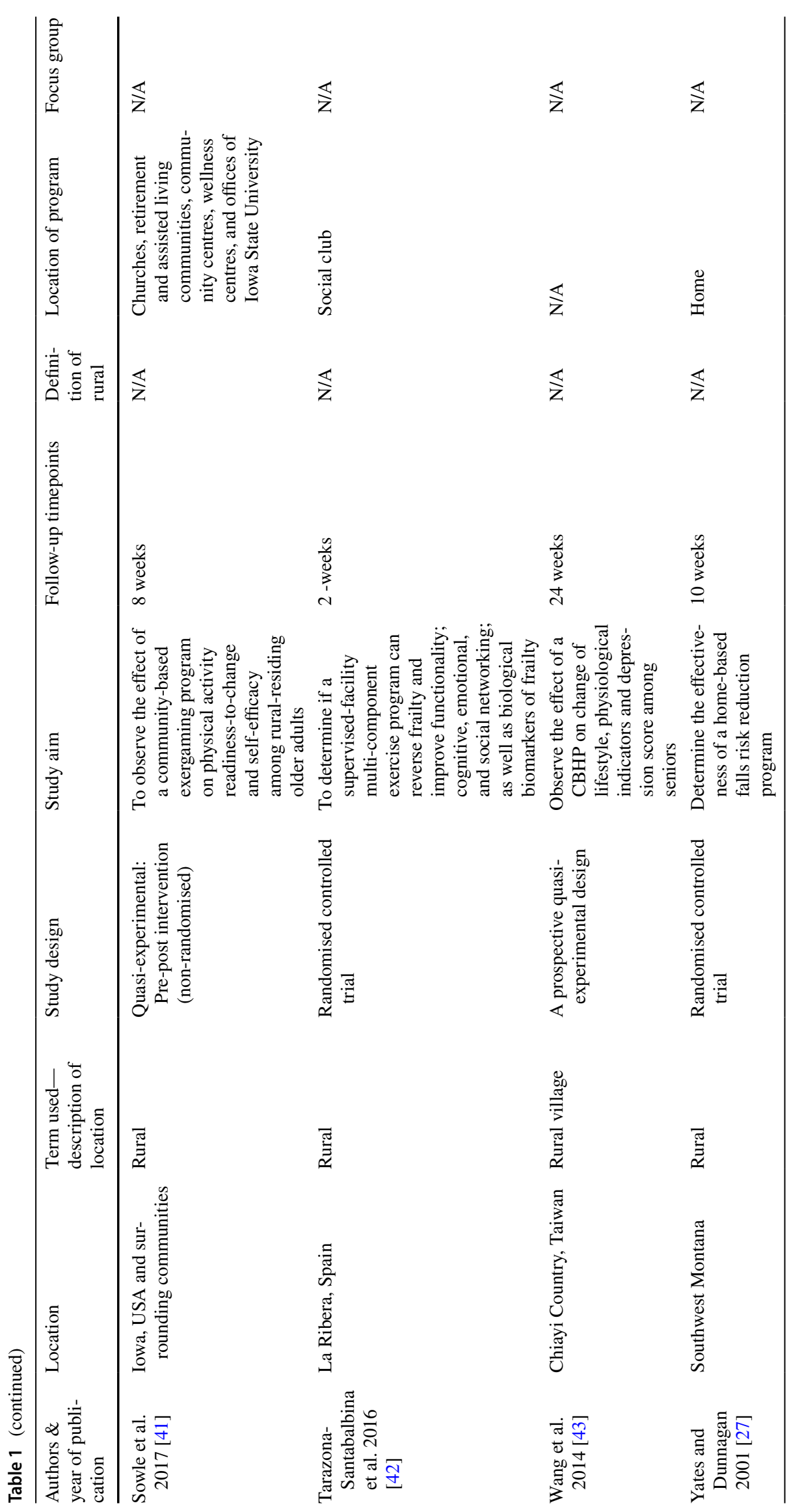


Table 2 The characteristics of the sample population for included studies

\begin{tabular}{|c|c|c|c|c|c|c|}
\hline \multirow{3}{*}{$\begin{array}{l}\text { Authors \& } \\
\text { year of publi- } \\
\text { cation }\end{array}$} & \multicolumn{6}{|c|}{ Sample characteristics } \\
\hline & \multirow[t]{2}{*}{ Sample size } & \multicolumn{2}{|l|}{ Age range (mean) } & \multicolumn{2}{|c|}{$\%$ Female $(n=)$} & \multirow[t]{2}{*}{ Chronic disease } \\
\hline & & Intervention & Comparison & Intervention & Comparison & \\
\hline $\begin{array}{l}\text { Jang et al. } \\
2018[33]\end{array}$ & $22($ Control $=11)$ & $66-70(68.6)$ & $68-76(72.5)$ & $17 \%(3)$ & $45 \%(5)$ & $\begin{array}{l}\text { None reported-although } \\
\text { included prefrail group } \\
\text { screened using the cardiovas- } \\
\text { cular health frailty phenotype } \\
\text { criteria }\end{array}$ \\
\hline $\begin{array}{l}\text { Jindo et al. } \\
2016[34]\end{array}$ & $68($ Control $=34)$ & $67-73(70.0)$ & $66-74(70.0)$ & $91.2 \%(31)$ & $91.2 \%(31)$ & $\begin{array}{l}\text { None reported-ambulant and } \\
\text { living independently }\end{array}$ \\
\hline $\begin{array}{l}\text { Jindo et al. } \\
2017 \text { [35] }\end{array}$ & 46 & $66.6-73.6(70.1)$ & N/A & $87 \%(40)$ & N/A & $\begin{array}{l}\text { None reported-ambulant and } \\
\text { living independently }\end{array}$ \\
\hline $\begin{array}{l}\text { Lin et al. } 2006 \\
{[36]}\end{array}$ & 88 & $\begin{array}{l}65+\text { (upper range } \\
\text { and mean not } \\
\text { specified) }\end{array}$ & N/A & $68.2 \%(60)$ & N/A & $\begin{array}{l}\text { Yes, } 68.2 \% \text { reported at least } \\
1 \text { comorbid condition (not } \\
\text { specified) }\end{array}$ \\
\hline $\begin{array}{l}\text { McMahon } \\
\text { et al. } 2016 \\
\text { [37] }\end{array}$ & $30($ Control $=14)$ & $78.9-88.3(83.6)$ & $78.9-88.3(83.6)$ & $93.3 \%(15)$ & $93.3 \%(13)$ & None reported \\
\hline $\begin{array}{l}\text { Muscari et al. } \\
2010[38]\end{array}$ & $120($ Control $=60)$ & $66.3-71.3(68.8)$ & $66.8-72.4(69.6)$ & $47.7 \%(28)$ & $50 \%(30)$ & $\begin{array}{c}5 \% \text { COPD, } 73.3 \% \text { Hypertension, } \\
60.8 \% \text { Hypercholesterolaemia }\end{array}$ \\
\hline $\begin{array}{l}\text { Okumiya et al. } \\
1996 \text { [39] }\end{array}$ & $42($ Control $=21)$ & $75-87(78.8)$ & $75-87(78.8)$ & $57.1 \%(12)$ & $57.1 \%(12)$ & None reported \\
\hline $\begin{array}{l}\text { Snapp et al. } \\
2009[40]\end{array}$ & $19($ Control $=9)$ & $>65(85)$ & $>65(85)$ & $80 \%(8)$ & $100 \%(9)$ & $\begin{array}{l}\text { None reported-Participants } \\
\text { living at an assisted living } \\
\text { facility or an independent liv- } \\
\text { ing facility }\end{array}$ \\
\hline $\begin{array}{r}\text { Sowle et al. } \\
2017 \text { [41] }\end{array}$ & 265 & $65-85+$ & N/A & $83.4 \%(221)$ & N/A & $\begin{array}{l}\text { None reported-ambulant and } \\
\text { living independently }\end{array}$ \\
\hline $\begin{array}{l}\text { Tarazona- } \\
\text { Santabalbina } \\
\text { et al. } 2016 \\
\text { [42] }\end{array}$ & $100($ Control $=49)$ & $76.1-83.3(79.7)$ & $76.6-84(80.3)$ & $56.9 \%(29)$ & $51 \%(25)$ & $\begin{array}{l}\text { 77\% Hypertension, } 46 \% \text { Hyper- } \\
\text { lipidaemia, } 8 \% \text { COPD }\end{array}$ \\
\hline $\begin{array}{l}\text { Wang et al. } \\
2014[43]\end{array}$ & $\begin{array}{r}520 \text { (Group } \\
\left.1^{*}=431\right)\end{array}$ & $68.53-80.81(74.67)$ & $71.45-82.01(76.73)$ & $65 \%(280)$ & $68.5 \%(61)$ & None reported \\
\hline $\begin{array}{l}\text { Yates and } \\
\text { Dunnagan } \\
2001[27]\end{array}$ & $37($ Control $=19)$ & $67-90(76)$ & $69-88(78)$ & $72.2 \%(13)$ & $68.4 \%(13)$ & None reported \\
\hline
\end{tabular}

*Group 1 = nursing home group intervention vs Group 2 = community-dwelling intervention

studies reported dietary outcomes, looking at a healthy diet and nutrition.

\section{Discussion}

The overall aim of this scoping review was to investigate the characteristics and outcomes of community-based exercise programs for older adults living in rural/regional areas. Only 12 publications met the predetermined eligibility criteria, indicating the lack of research in the rural/regional context as well as the lack of qualitative/psychosocial outcomes.
Approximately one-third of older adults live outside of major cities in Australia, Canada, and United States [44-46], whereby access to health services and community exercise programs is often limited due to access, travel, and affordability. Highlighting the importance of specific consideration for the needs of the rural/regional older adult population.

Achieving healthy ageing requires adequate healthcare systems; an integral part of which includes having long-term exercise and physical activity programs in place to prevent a litany of chronic and other diseases, and thus be proactive in improving the health and wellbeing of an increasing ageing population throughout the world [9] Indeed, Izquirdo et al. 


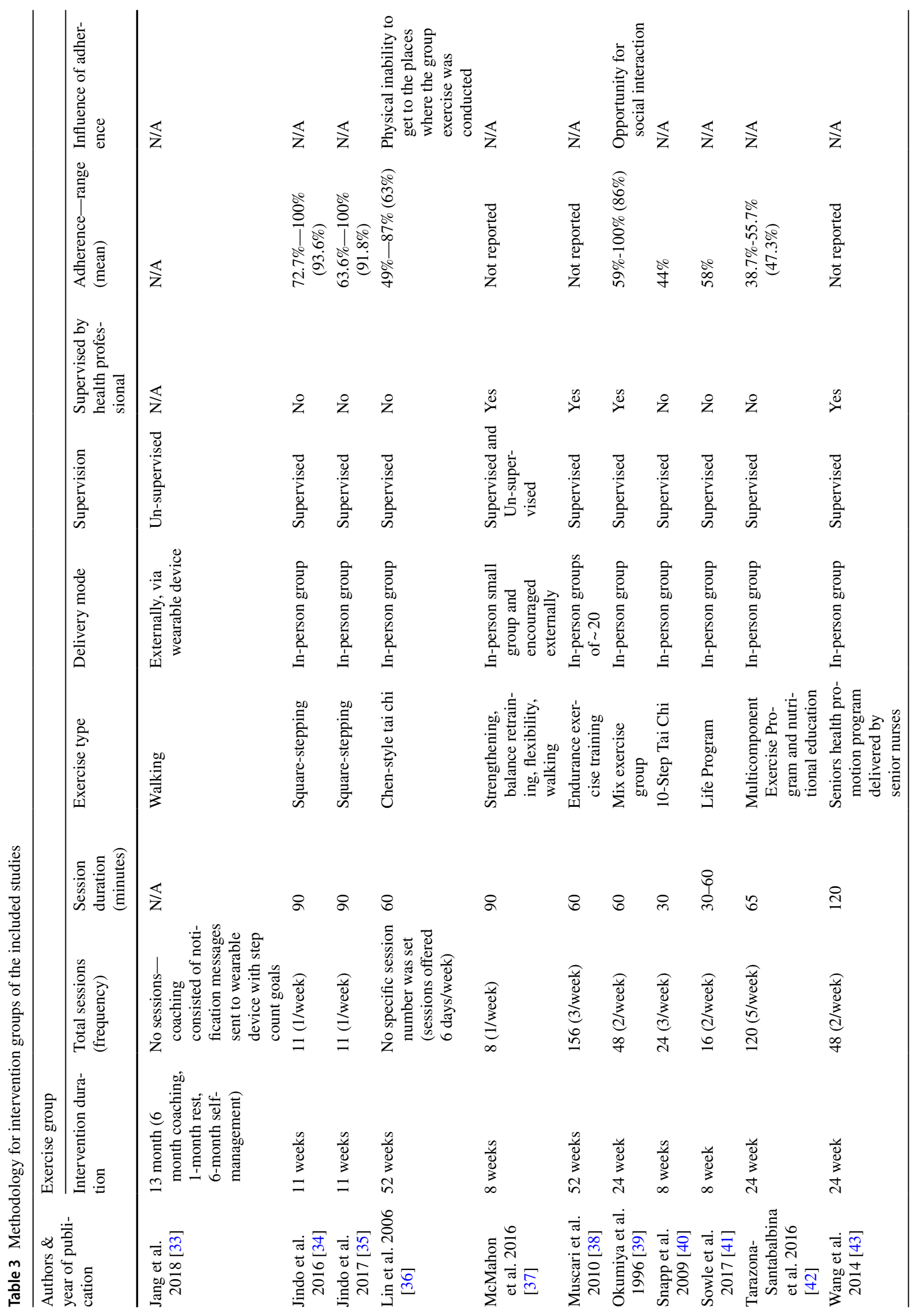




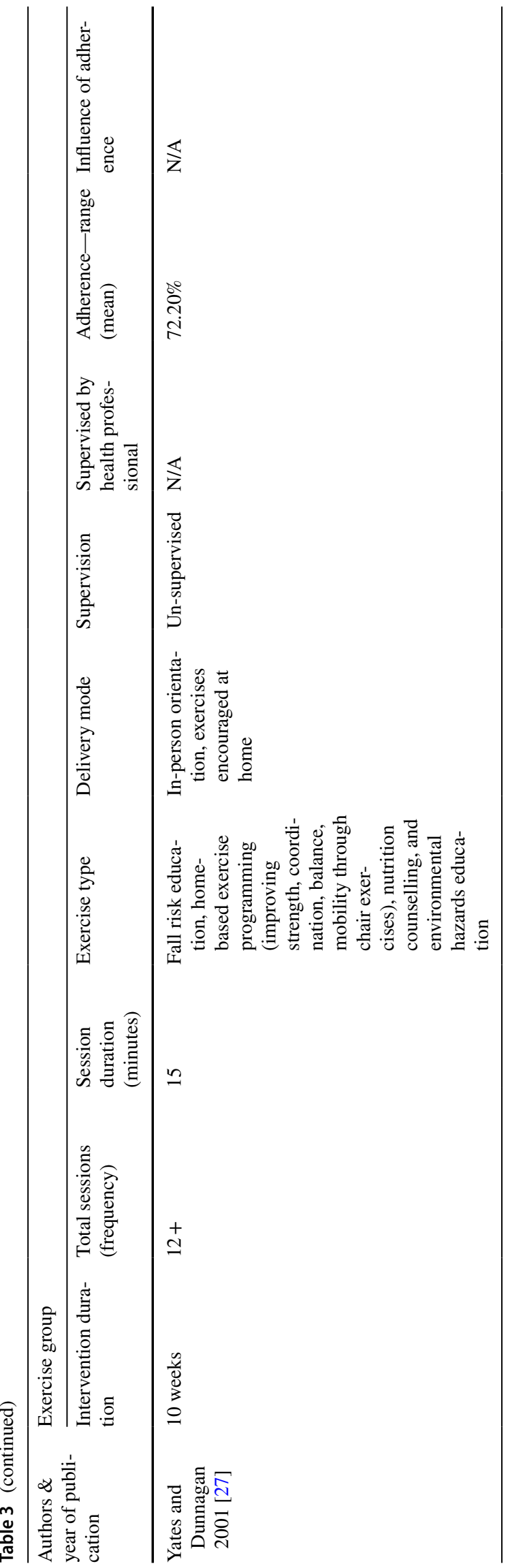

(2021) suggest that the current body of evidence makes it 'unethical' not to prescribe physical activity to older adults. There is an opportunity to address the inequity of health in rural/regional older adults by offering accessible forms of exercise. This scoping review has provided an opportunity to assess the characteristics of community-based exercise programs that are likely to be feasible and effective in rural/ regional settings.

All interventions in the current review included low-to moderate intensity exercise; predominantly walking, squarestepping and Tai Chi. This finding is consistent with the systematic review by Moore et al. [22]. However, Moore and colleagues included studies from urban and rural communities (two out of the seven studies) [22], whereas the current review was specifically focussed on rural/regional communities. Vast variety in the type, delivery and duration of exercise interventions was found across the included studies. Most programs (72\%) offered only a single exercise type, and the studies largely evaluated physical function (83\%) rather than psychological measures (50\%). Interestingly, a review of 19 systematic reviews relating to exercise interventions for older adults living in the community revealed that any modality of exercise whether multi-modal or single, were more effective at increasing physical activity levels than no intervention at all [47]. Regardless of the setting, community-based exercise programs should include a variety of multi-component exercises of a moderate intensity, both to align the program with WHO 2020 guidelines on physical activity and sedentary behaviour [48] and to provide the participants with variety and autonomy/choice. Of particular interest, in line with WHO 2020 guidelines on physical activity [48], 150-300 min of moderate intensity or 75-150 min of vigorous intensity was prescribed in four studies [38, 42, 43, 49] as referred to in Table 4. Alongside the WHO exercise guidelines, a strong recommendation for older adults to take part in multi-component exercise that includes functional balance and strength training on three or more days a week to reduce the risk of falls is also included [48]. Only two of those previous four studies met this additional requirement $[38,42]$.

A large proportion of these studies included in this review pre-date the use of video conferencing platforms. However, with the spread of COVID-19, the use of video teleconferencing for the delivery of exercise interventions to rural/ regional areas have not yet been capitalised for the use of telehealth and online fitness programs [50]. Whilst telehealth does increase falls risk as supervision cannot assist participants if they are to lose their balance, there is still the need for older adults to participate in exercise to improve quality of life, functional independence, and health [51]. Telehealth exercise interventions may also be considered a positive for rural/regional areas, where access to professional services / 


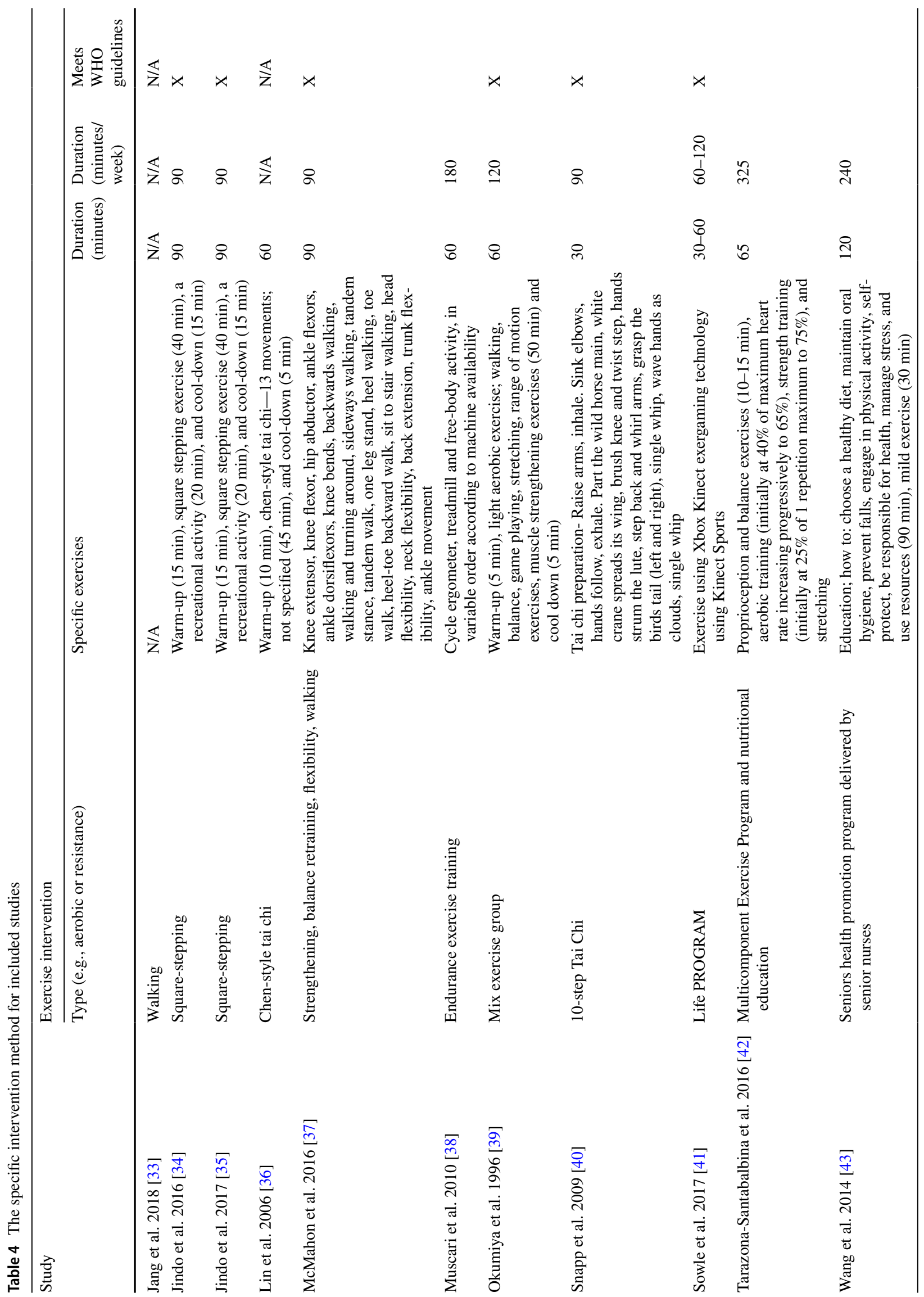




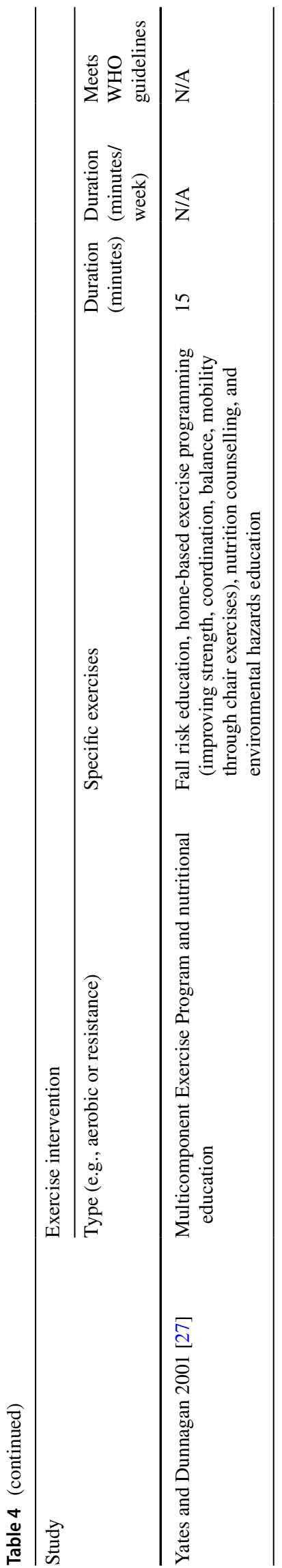

supervision is thus improved, the need for travel is reduced, and less strain is placed on the healthcare system [51].

Consistent with two previous reviews, a key finding from this review was the limited number of studies that utilised valid and reliable measurement tools to measure the outcomes (e.g., functional outcomes) to quantify the benefits of community-based exercise interventions. In terms of functional outcomes, the use of valid and objective measures of functional ability (such as $30 \mathrm{~s}$ chair sit to stand, timed up and go, and a balance test) in combination with at least one validated body composition measurement, a psychosocial outcome, and a cognitive measurement would help to provide consistency with reporting of important outcomes. The main outcomes measured and reported were functional outcomes, which were reported in 10 of 12 studies. Whilst psychosocial, body composition, and cardiovascular health outcomes were also reported, the lack of qualitative investigation of psychosocial parameters known to influence health outcomes, such as loneliness, and the lack of assessment of other health-related behaviours such as dietary patterns, limits the conclusions that can be drawn about the effectiveness of exercise interventions for improving health outcomes of older adults in rural/regional areas.

Healthy ageing is multi-faceted and requires a holistic focus on all aspects of health and wellbeing, including functional, body composition, psychosocial, cardiovascular, and dietary outcomes. However, none of the published programs evaluated all the relevant measurable outcomes. Noting that none of the included studies evaluated a measurable outcome of social participation or loneliness. Loneliness reduces quality of life and is associated with a $29 \%$ increased risk of mortality and higher chronic disease occurrence [16]. Exercise can facilitate social participation; however, it may be limited in its reach among older adults living in rural/ regional areas, potentially increasing health inequalities and vulnerability of this sector of the population [52]. In turn, by not placing exercise as an important factor in the model of healthy ageing, significant limitations are being imposed on older adults' independence and quality of life [53, 54].

Overall, there is still a limited understanding of the characteristics and effectiveness of community-based exercise programs, particularly within rural/regional areas. These areas face unique challenges, such as limited access to equipment/resources, transportation, and services, as well as significant costs to run the programs, which are important considerations in the success of programs. In addition, outcome measures should be valid, reliable, and applicable to regional older adult populations and factors associated with wellbeing, longevity, and quality of life. This would enable a greater understanding of the clinical importance and real-life impact of an intervention or exercise program in areas of need. 


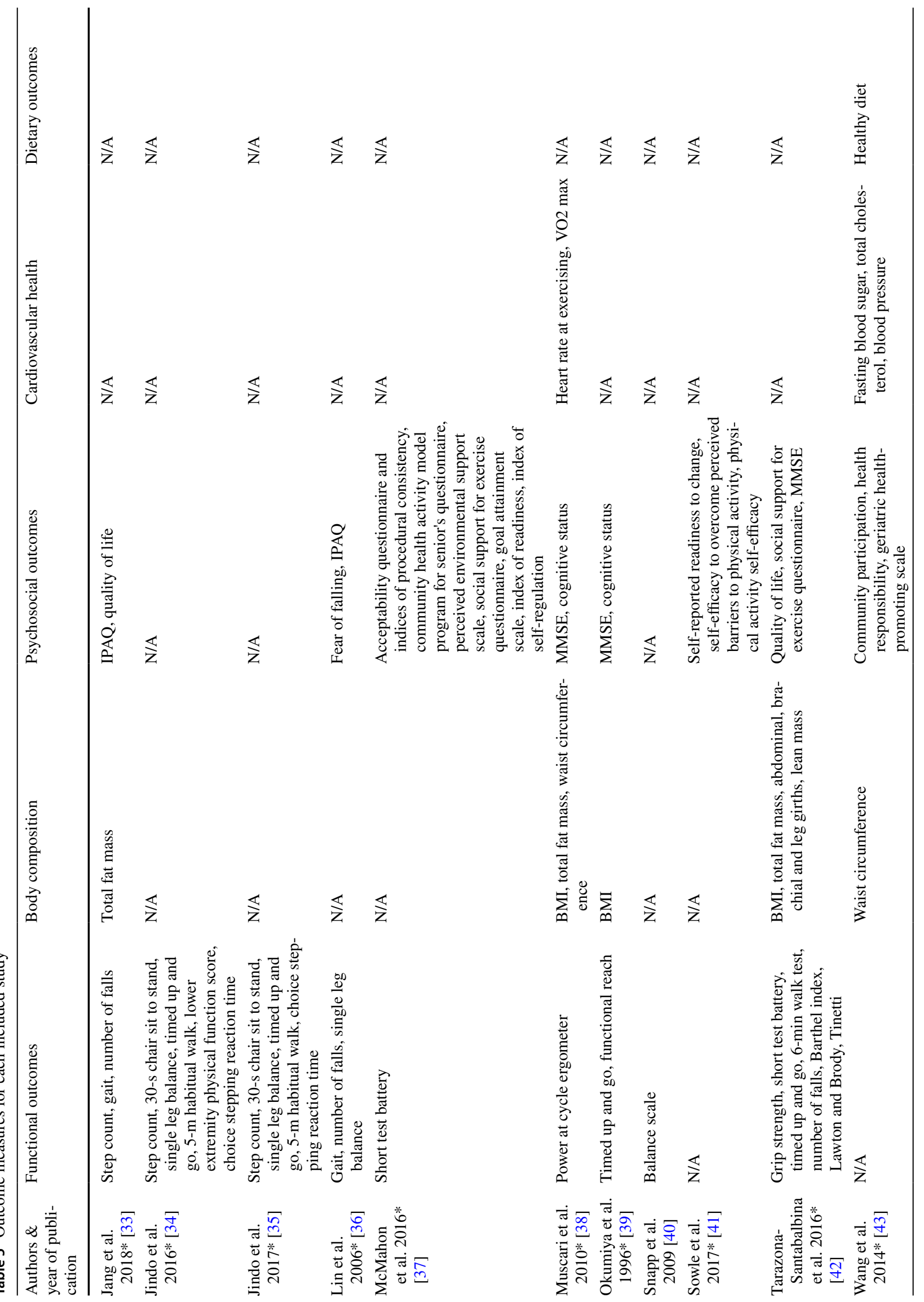


Figure 2 presents recommendations from the included studies integrated with the authors' suggestions for successful community-based exercise program delivery for older adults residing in rural/regional areas.

\section{Recommendations for future research}

Future research is essential to investigate the effectiveness, acceptability, and successful implementation of community-based exercise interventions located in rural/regional areas. Of note, a significant finding of this review was that no studies clearly defined a rural/regional location. The assumption that a program is based in a rural/regional area based on minimal reported details, limits the specificity of the recommendations to programs running in rural/ regional areas. Future research should include the location details of programs, particularly given the dynamics of program success that may be impacted with geographical location. We recommend that a definition of a rural/ regional area is utilised.

In addition, future research must place strong consideration on participant recruitment to ensure representative samples, as most studies were limited by small sample sizes, high percentage of female participants, selection bias, exclusion of frailer older adults or those with dementia, and no blinding. More representative sampling will inform the development of relevant recommendations aimed at improving community-based exercise programs for older adults that consider the diversity of populations in regional/rural areas.

Finally, psychosocial outcomes were sparsely reported in study outcomes. Exercise can have profound psychosocial impacts on older adults [12, 55], whilst consideration of such behavioural elements can enhance program adherence, increasing participant motivation, self-efficacy and feelings of control $[16,56]$. Such psychosocial improvements can in-turn lead to increasing general health, physical activity, social interaction and independence, concurrently enhancing mental wellbeing and Quality of Life [57]. Considering the pivotal impact that psychosocial factors such as wellbeing and depression have on the physical function and quality of life of older adults [58], the current authors strongly recommend that future research addresses this gap in the current literature.

\section{Limitations and gaps}

This review may not contain an exhaustive list of community-based exercise interventions that may benefit the physical and psychosocial wellbeing of older adults living in rural/regional area. This study only investigated rural/ regional community-based exercise interventions targeting 


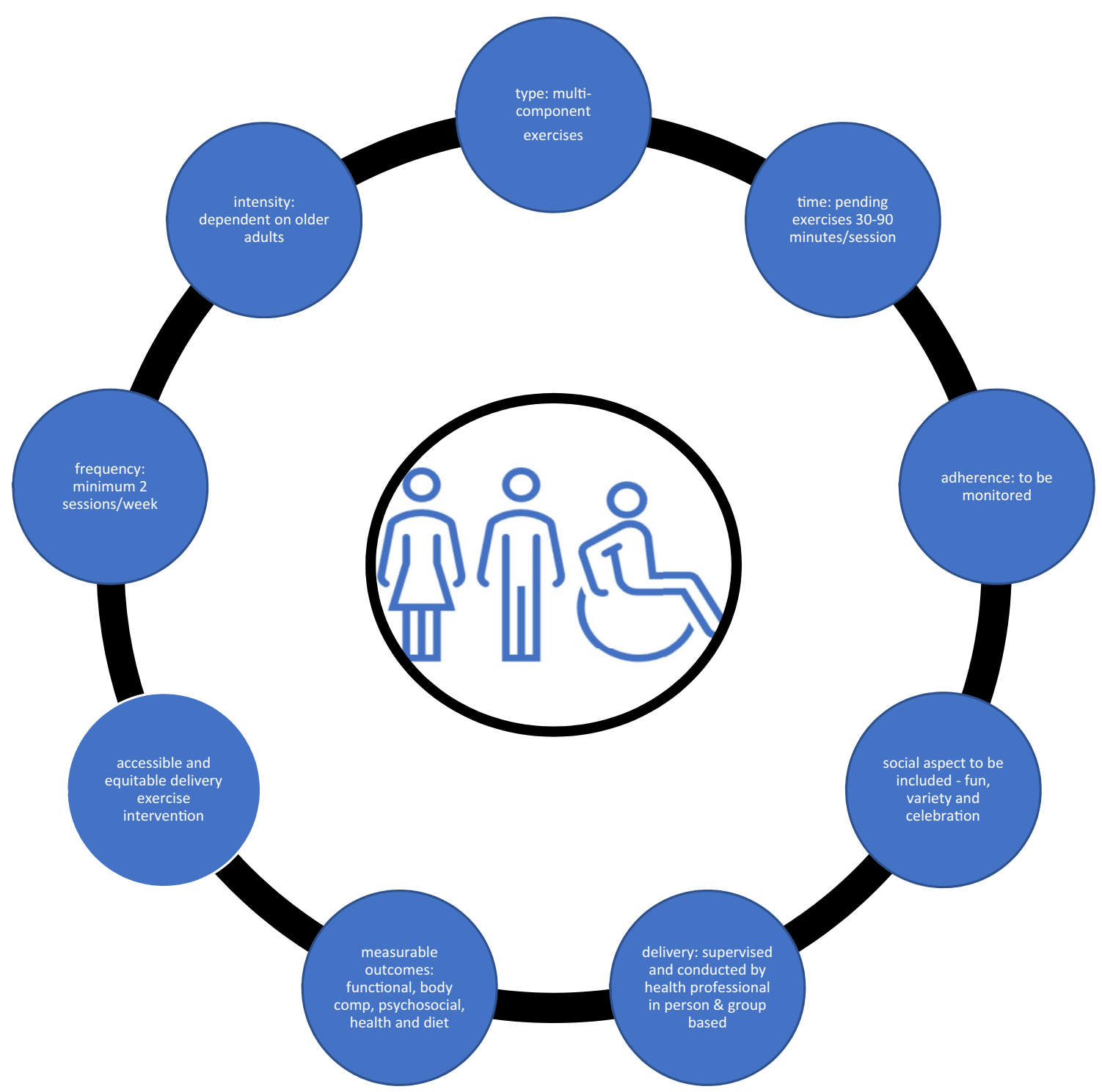

Fig. 2 Variables to include in a successful rural/regional community-based exercise programs

older adults aged 65 years or older. As stated by Moore et al. [22] there is currently no international definition of 'older adults' and as stated by the UN there is currently no international definition for rural/regional areas [59]. Therefore, limiting our search to adults aged over 65 years and studies that explicitly stated by the authors in rural/regional areas studies including populations that may have been considered older in certain locations may have been overlooked [22]. Studies should specifically identify 'rural/regional settings' as a focus, and to make this clearer as to the location of their study. There is a real need for more focused research that contrasts the needs of regional versus non-regional communities where one approach may not be suitable for all participants.
Several studies did not specify what target population was being included in the study or included those below the age of 65 years; and/or did not include a comparison group as a control group or of a lesser intensity than the exercise group. This highlights the need for researchers to clearly report characteristics of intervention participants and to include a control/comparison group when undertaking evaluations of community-based exercise interventions or programs in rural/regional areas.

Most studies in this review reported at least one limitation to their findings (88\%); only two studies did not report on any limitations (12\%). Most commonly, small sample size was noted as a limitation, reported in seven studies (39\%). Other common limitations included lack of a control group (28\%), not accounting for potential confounding 
variables such as social/psychological factors (28\%) and poor study design (28\%). These limitations present exciting and important avenues for future investigation in this area.

\section{Conclusion}

Evaluation of the effectiveness of rural/regional communitybased exercise programs was primarily focused on physical and functional health outcomes, with almost $90 \%$ of included studies reporting such measures. Low male representation was common, with women outnumbering men in most studies, presenting the need for recruitment strategies that target men as well as women. Finally, there has been minimal investigation of qualitative outcomes in existing community-based exercise programs, presenting a key gap for future research to address. The importance of effective community-based exercise programs is vital for the physical and psychosocial health of older adults living in rural/ regional locations in an ageing international climate.

Supplementary Information The online version contains supplementary material available at https://doi.org/10.1007/s40520-022-02079-y.

Acknowledgements Roger Carter, Liaison Librarian, Library, University of the Sunshine Coast, Sippy Downs, Australia. Roger provided assistance with the search strategy. Dana Craven for her assistance with the screening as the third reviewer as well as designing the study.

Author contributions JM, CA, DW, MS, and HS designed the study. JM and SF performed the search. CL completed data extraction. SF, CL, CA, JM, and MS wrote the manuscript. All authors contributed to the critical revision of the manuscript.

Funding Open Access funding enabled and organized by CAUL and its Member Institutions. This study was supported by a University of the Sunshine Coast-Sunshine Coast Council Regional Partnership Agreement Grant.

Availability of data and material All data used for this review are presented in tables, figures, or supplementary files. Further queries can be submitted to the corresponding author.

Code availability Not applicable.

\section{Declarations}

\section{Conflict of interest None.}

Ethics approval This review does not contain any experiments involving human participants or animals performed by any of the authors.

Consent to participate For this type of study, informed consent forms were not required.
Open Access This article is licensed under a Creative Commons Attribution 4.0 International License, which permits use, sharing, adaptation, distribution and reproduction in any medium or format, as long as you give appropriate credit to the original author(s) and the source, provide a link to the Creative Commons licence, and indicate if changes were made. The images or other third party material in this article are included in the article's Creative Commons licence, unless indicated otherwise in a credit line to the material. If material is not included in the article's Creative Commons licence and your intended use is not permitted by statutory regulation or exceeds the permitted use, you will need to obtain permission directly from the copyright holder. To view a copy of this licence, visit http://creativecommons.org/licenses/by/4.0/.

\section{References}

1. Maresova P, Javanmardi E, Barakovic S et al (2019) Consequences of chronic diseases and other limitations associated with old age - a scoping review. BMC Public Health 19:1431. https://doi.org/10.1186/s12889-019-7762-5

2. Australian Institute of Health and Welfare (2020) Burden of disease. Retrieved from https://www.aihw.gov.au/reports-data/ health-conditions-disability-deaths/burden-of-disease/overview

3. Hajat C, Stein E (2018) The global burden of multiple chronic conditions: a narrative review. Preventitive Med Rep 12:284293. https://doi.org/10.1016/j.pmedr.2018.10.008

4. Ng C, Fairhall N, Wallbank G et al (2019) Exercise for falls prevention in community-dwelling older adults: trial and participant characteristics, interventions and bias in clinical trials from a systematic review. BMJ Open Sport Exerc Med 5:e000663. https://doi.org/10.1136/bmjsem-2019-000663

5. Scott AJ, Ellison M, Sinclair DA (2021) The economic value of targeting aging. Nature Aging 1:616-623. https://doi.org/10. 1038/s43587-021-00080-0

6. National Centre for Chronic Disease Prevention and Health Promotion (CDC) (2021) Centres for Disease Control and Prevention. Retrieved from https://www.cdc.gov/chronicdisease/about/ index.htm.

7. World Health Organisation (2018) Ageing and health. Retrieved from https://www.who.int/news-room/fact-sheets/detail/ ageing-and-health.

8. Thomas SL, Wakerman J, Humphreys JS (2015) Ensuring equity of access to primary health care in rural and remote Australia - what core services should be locally available? Int J Equity Health 14:111. https://doi.org/10.1186/s12939-015-0228-1

9. World Health Organisation (2015) Wolrd Report on Ageing and Health. Retrieved from: https://apps.who.int/iris/handle/10665/ 186463.

10. Di Lorito C, Long A, Byrne A et al (2021) Exercise interventions for older adults: A systematic review of meta-analyses. J Sport Health Sci 10:29-47. https://doi.org/10.1016/j.jshs.2020. 06.003

11. Lindsay Smith G, Banting L, Eime R et al (2017) The association between social support and physical activity in older adults: a systematic review. Int J Behav Nutr Phys Act 14:56. https:// doi.org/10.1186/s12966-017-0509-8

12. Mandolesi L, Polverino A, Montuori S et al (2018) Effects of physical exercise on cognitive functioning and wellbeing: biological and psychological benefits. Front Psychol 9:509-509. https://doi.org/10.3389/fpsyg.2018.00509

13. Hannan M, Cheng D, Green E et al (2004) Establishing the compliance in elderly women for use of a low level mechanical stress device in a clinical osteoporosis study. Osteoporos Int 15:918-926. https://doi.org/10.1007/s00198-004-1637-y 
14. Warburton D, Bredin S (2017) Health benefits of physical activity: a systematic review of current systematic reviews. Curr Opin Cardiol 32:541-556. https://doi.org/10.1097/HCO.0000000000 000437

15. World Health Organisation (2020a) Physical activity. Retrieved from https://www.who.int/news-room/fact-sheets/detail/physi cal-activity.

16. Izquierdo M, Duque G, Morley JE (2021) Physical activity guidelines for older people: knowledge gaps and future directions. Lancet Healthy Longevity 2:e380-e383. https://doi.org/ 10.1016/S2666-7568(21)00079-9

17. Seo JY, Chao YY (2018) Effects of exercise interventions on depressive symptoms among community-dwelling older adults in the united states: a systematic review. J Gerontol Nurs 44:31-38. https://doi.org/10.3928/00989134-20171024-01

18. Shankar A, McMunn A, Banks J et al (2011) Loneliness, social isolation, and behavioral and biological health indicators in older adults. Health Psychol 30:377-385. https://doi.org/10.1037/a0022 826

19. Stickley A, Koyanagi A (2018) Physical multimorbidity and loneliness: A population-based study. PLoS ONE 13:e0191651. https://doi.org/10.1371/journal.pone.0191651

20. de Vries NM, van Ravensberg CD, Hobbelen JS et al (2012) Effects of physical exercise therapy on mobility, physical functioning, physical activity and quality of life in community-dwelling older adults with impaired mobility, physical disability and/ or multi-morbidity: a meta-analysis. Ageing Res Rev 11:136-149. https://doi.org/10.1016/j.arr.2011.11.002

21. Frost R, Belk C, Jovicic A et al (2017) Health promotion interventions for community-dwelling older people with mild or pre-frailty: a systematic review and meta-analysis. BMC Geriatr 17:157. https://doi.org/10.1186/s12877-017-0547-8

22. Moore M, Warburton J, O'Halloran PD et al (2016) Effective community-based physical activity interventions for older adults living in rural and regional areas: a systematic review. J Aging Phys Act 24:158-167. https://doi.org/10.1123/japa.2014-0218

23. Devereux K, Robertson D, Briffa NK (2005) Effects of a waterbased program on women 65 years and over: a randomised controlled trial. Aust J Physiother 51:102-108. https://doi.org/10. 1016/S0004-9514(05)70038-6

24. Pahor M, Guralnik JM, Ambrosius WT et al (2014) Effect of structured physical activity on prevention of major mobility disability in older adults: The life study randomized clinical trial. J Am Med Assoc 311:2387-2396. https://doi.org/10.1001/jama. 2014.5616

25. Petrella RJ, Koval JJ, Cunningham DA et al (2003) Can primary care doctors prescribe exercise to improve fitness? The Step Test Exercise Prescription (STEP) project. Am J Prev Med 24:316322. https://doi.org/10.1016/S0749-3797(03)00022-9

26. Sharpe PA, Jackson KL, White C et al (1997) Effects of a oneyear physical activity interven- tion for older adults at congregate nutrition sites. Gerontologist 37:208-215. https://doi.org/10.1093/ geront/37.2.208

27. Yates SM, Dunnagan TA (2001) Evaluating the effectiveness of a home-based fall risk reduction program for rural communitydwelling older adults. J Gerontol Series A Biol Sci Med Sci 56:M226-M230. https://doi.org/10.1093/gerona/56.4.m226

28. Osho O, Owoeye O, Armijo-Olivo S (2018) Adherence and attrition in fall prevention exercise programs for community-dwelling older adults: a systematic review and meta-analysis. J Aging Phys Act 26:304-326. https://doi.org/10.1123/japa.2016-0326

29. Holt-Lunstad J, Smith TB, Baker M et al (2015) Loneliness and social isolation as risk factors for mortality:na meta-analytic review. Perspect Psychol Sci 10(2), 227-237. https://journals. sagepub.com/doi/abs/https://doi.org/10.1177/1745691614568352
30. Munn Z, Peters MDJ, Stern C et al (2018) Systematic review or scoping review? Guidance for authors when choosing between a systematic or scoping review approach. BMC Med Res Methodol 18:143. https://doi.org/10.1186/s12874-018-0611-x

31. Peters MD J, Godfrey C, McInerney P et al (2020) Chapter 11: Scoping Reviews (JBI Reviewer's Manual. JBI. Ed.)

32. Tricco AC, Lillie E, Zarin W et al (2018) PRISMA Extension for scoping reviews (PRISMA-ScR): checklist and explanation. Ann Intern Med 169:467-473. https://doi.org/10.7326/m18-0850\% m30178033

33. Jang IY, Kim HR, Lee E et al (2018) Impact of a wearable devicebased walking programs in rural older adults on physical activity and health outcomes: Cohort study. JMIR mHealth uHealth. https://doi.org/10.2196/11335

34. Jindo T, Tsunoda K, Kitano N et al (2016) Pedometers affect changes in lower-extremity physical function during a squarestepping exercise program in older Japanese adults. J Geriatric Phys Ther 39:83-88. https://doi.org/10.1519/JPT.0000000000 000054

35. Jindo T, Kitano N, Tsunoda K et al (2017) Daily life physical activity modulates the effects of an exercise program on lowerextremity physical function in japanese older adults. J Geriat Phys Ther 40:150-157. https://doi.org/10.1519/JPT.0000000000 000087

36. Lin MR, Hwang HF, Wang YW et al (2006) Community-based tai chi and its effect on injurious falls, balance, gait, and fear of falling in older people. Phys Ther 86:1189-1201. https://doi.org/ $10.2522 / \mathrm{ptj} .20040408$

37. McMahon SK, Wyman JF, Belyea MJ et al (2016) Combining motivational and physical intervention components to promote fall-reducing physical activity among community-dwelling older adults: a feasibility study. Am J Health Promot AJHP 30:638-644. https://doi.org/10.4278/ajhp.130522-ARB-265

38. Muscari A, Giannoni C, Pierpaoli L et al (2010) Chronic endurance exercise training prevents aging-related cognitive decline in healthy older adults: A randomized controlled trial. Int J Geriatr Psychiatry 25:1055-1064. https://doi.org/10.1002/gps.2462

39. Okumiya K, Matsubayashi K, Wada T et al (1996) Effects of exercise on neurobehavioral function in community-dwelling older people more than 75 years of age. J Am Geriatr Soc 44:569-572. https://doi.org/10.1111/j.1532-5415.1996.tb01444.x

40. Snapp BR, Malkin MJ, Lloyd LF et al (2009) Effects of modified Tai Chi Chuan on balance in older adults. Am J Recreat Ther 8:25-30. https://doi.org/10.5055/ajrt.2009.0018

41. Sowle AJ, Francis SL, Margrett JA et al (2017) A communitybased exergaming physical activity program improves readinessTo-change and self-efficacy among rural-residing older adults. J Aging Phys Act 25:432-437. https://doi.org/10.1123/japa. 2015-0278

42. Tarazona-Santabalbina FJ, Gómez-Cabrera MC, Pérez-Ros $P$ et al (2016) A multicomponent exercise intervention that reverses frailty and improves cognition, emotion, and social networking in the community-dwelling frail elderly: a randomized clinical trial. $\mathrm{J}$ Am Med Dir Assoc 17:426-433. https://doi.org/10.1016/j.jamda. 2016.01.019

43. Wang J, Chen CY, Lai LJ et al (2014) The effectiveness of a community-based health promotion program for rural elders: a quasi-experimental design. Appl Nurs Res 27:181-185. https:// doi.org/10.1016/j.apnr.2013.11.014

44. Australian Bureau Statistics (2018) Older Australia at a glance. Retrieved from: https://www.aihw.gov.au/reports/older-people/ older-australia-at-a-glance/contents/diverse-groups-of-older-austr alians/regional-remote-communities

45. Turcotte M, Schellenberg G (2007) A portrait of seniors in Canada 2006. Ottawa: Statistics Canada; 2007. Retrieved from https:// 
www150.statcan.gc.ca/n1/pub/89-519-x/89-519-x2006001-eng. pdf

46. United States Census Bureau (2019) The older population in rural america: 2012-2016. Retrieved from: https://www.census.gov/ library/publications/2019/acs/acs-41.html Retrieved from

47. Zubala A, MacGillivray S, Frost $\mathrm{H}$ et al (2017) Promotion of physical activity interventions for community dwelling older adults: A systematic review of reviews. PLoS ONE 12:e0180902e0180902. https://doi.org/10.1371/journal.pone.0180902

48. World Health Organisation (2020b) WHO Guidelines on physical activity and sedentary behaviour. Retrieved from https://www. who.int/publications/i/item/9789240015128. Retrieved from

49. Okubo Y, Osuka Y, Jung S et al (2016) Walking can be more effective than balance training in fall prevention among community-dwelling older adults. Geriatr Gerontol Int 16:118-125. https://doi.org/10.1111/ggi.12444

50. Fisk M, Livingstone A, Pit SW (2020) Telehealth in the context of covid-19: changing perspectives in Australia, the United Kingdom, and the United States. J Med Internet Res 22:e19264. https:// doi.org/10.2196/19264

51. Middleton A, Simpson KN, Bettger JP et al (2020) COVID-19 pandemic and beyond: considerations and costs of telehealth exercise programs for older adults with functional impairments living at home-lessons learned from a pilot case study. Phys Ther 100:1278-1288. https://doi.org/10.1093/ptj/pzaa089

52. Egan M, Tannahill C, Petticrew M et al (2008) Psychosocial risk factors in home and community settings and their associations with population health and health inequalities: a systematic meta-review. BMC Public Health 8:239. https://doi.org/10.1186/ 1471-2458-8-239
53. Eklund K, Wilhelmson K (2009) Outcomes of coordinated and integrated interventions targeting frail elderly people: a systematic review of randomised controlled trials. Health Soc Care Commun 17:447-458. https://doi.org/10.1111/j.1365-2524.2009.00844.x

54. Low L-F, Yap M, Brodaty H (2011) A systematic review of different models of home and community care services for older persons. BMC Health Serv Res 11:93. https://doi.org/10.1186/ 1472-6963-11-93

55. de Oliveira L, Souza EC, Rodrigues RAS et al (2019) The effects of physical activity on anxiety, depression, and quality of life in elderly people living in the community. Trends Psychiatry Psychother 41:36-42. https://doi.org/10.1590/2237-6089-2017-0129

56. Knobf MT, Musanti R, Dorward J (2007) Exercise and quality of life outcomes in patients with cancer. Semin Oncol Nurs 23:285296. https://doi.org/10.1016/j.soncn.2007.08.007

57. Antunes HKM, Stella SG, Santos RF et al (2005) Depression, anxiety and quality of life scores in seniors after an endurance exercise program. Brazilian J Psychiatry 27:266-271. https://doi. org/10.1590/S1516-44462005000400003

58. Pérez-Ros P, Martínez-Arnau FM, Tarazona-Santabalbina FJ (2019) Risk factors and number of falls as determinants of quality of life of community-dwelling older adults. J Geriatric Phys Ther 42:63-72. https://doi.org/10.1519/jpt.0000000000000150

59. United Nations (2017) Population density and urbanization. Retrieved from https://unstats.un.org/unsd/demographic/sconc erns/densurb/densurbmethods.htm

Publisher's Note Springer Nature remains neutral with regard to jurisdictional claims in published maps and institutional affiliations. 